\title{
The mechanics of superplastic forming - how to incorporate and model superplastic and superplastic-like conditions.
}

\author{
O.I. Bylya ${ }^{1,2, a}$, R.A. Vasin ${ }^{2, b}$ \& P.L. Blackwell ${ }^{3, c^{*}}$ \\ ${ }^{1}$ Institute of Technical Education and Research, SoA University, Bhubaneswar, India \\ ${ }^{2}$ Institute of Mechanics, Lomonosov Moscow State University, Moscow, Russia \\ ${ }^{3}$ AFRC, University of Strathclyde, Glasgow, UK

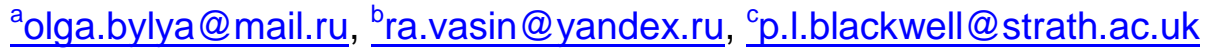

Keywords: Constitutive modelling, superplastic, visco-plastic, finite element

\begin{abstract}
Much work has been carried out in understanding the mechanics of superplasticity (SP). Some of the present challenges in SP forming revolve around the use of lower forming temperatures and faster strain rates, which may involve pushing the process boundaries to incorporate "superplastic-like" forming - perhaps also in materials with non-optimized microstructures. For process optimization there is a requirement to be able to model both within the SP and superplastic-like processing window in an integrated way. From a mechanics point of view the presence of high rate sensitivity is often seen as the key factor in controlling SP response. However, changes in phase distribution and grain morphology, or the accumulation of damage (cavitation) may compromise this assumption. The paper will examine the range of validity of some SP constitutive models and how they may be adapted to take into account processing routes that may incorporate superplastic-like and more conventional SP deformation modes.
\end{abstract}

\section{Introduction}

Superplastic forming (SPF) is noted as being an expensive manufacturing methodology. The high cost is linked to the relatively high forming temperatures and prolonged forming times. There have been a number of initiatives to improve this situation, including the use of ultrafine grained materials, modified alloy compositions and hybrid production techniques that combine an initial high strain rate deformation phase e.g. using hot drawing, with SPF [1, 2, 3]

This paper focuses on the latter approach which uses an initially high strain rate to generate most of the deformation in a component and then makes use of the superplastic (SP) properties of a material to generate the more detailed geometry elements of the final shape. For example, Guo et al [1] and Liu et al [2] used a hot deep drawing operation to impart significant strain to a Ti-6Al-4V part prior to finish forming using gas pressure at $800^{\circ} \mathrm{C}$ (this is lower than the optimal SP temperature of $900-920^{\circ} \mathrm{C}$ ). Through this hybrid process they managed to markedly reduce the forming time.

The underlying point in these various examples is that it is possible to potentially significantly reduce manufacturing time for a component by forming using a combination of what is often referred to as "superplastic-like" forming together with SP forming proper. In fact of course most conventional SPF operations will see some part of the sheet forming outside of the optimum SP window e.g. either due to temperature variations across the tooling, or due to variations in strain rate, especially during final shaping into corners.

From a process modelling point of view there is therefore a challenge to define a set of constitutive equations that can allow for this. There have of course been numerous attempts to develop models to represent material behaviour under SP conditions [4]. Many such models take either a power law or hyperbolic sine form [4, 5]. Such approaches, while valid within some particular range of temperature and strain rate, will however become invalid outside of the 
deformation regime associated with SP. Thus, a problem with many of the equations proposed is that they are not self-validating and often the equations used contain a range of parameters that are assumed to be constant e.g. $m$ itself, or some activation energy-like function $(Q)$.

In order to model a process that may involve deforming within the SP and non-SP field there is then a challenge to develop constitutive equations that operate over a range of deformation states i.e. under SP conditions where grain boundary sliding predominates and under faster rate non-SP conditions where slip will tend to be of greater significance. There have been previous attempts at this [2] that have used a crystal plasticity type approach and attempted to build models based on a range of factors including knowledge of slip mechanisms, vacancy concentrations, diffusion rates, back-stresses, etc. Such models are mathematically complex and require the determination of a wide range of parameters and though they may appear to be scientifically rigorous the fact is that they make many assumptions regarding the balance of deformation process operating within some particular deformation space. From a practical point of view it is questionable whether they offer any advantage over simpler approaches.

Another possibility is to use an equation set based around a phenomenological approach. In order to do this it becomes necessary to define the boundaries of the various regimes linked to SP. For the purposes of this paper it is assumed that the deformation is tensile and consideration is limited to metallic alloys. Usually, a plot of the natural logarithm of stress against strain rate is used to describe three regimes that could be used to define superplastic-like and SP deformation; Fig. 1a,3a. As is well known, the slope of this figure may be used to measure the strain rate sensitivity parameter, $m$, Fig. 1b. Generally, an $m$ of 0.3 or above might be considered to define the region of true SP. However, in practice factors such as cavitation e.g. in aluminium alloys, may compromise this.

Based on the above comments superplastic-like would primarily refer to a high temperature deformation process that involves some relatively high extension of the workpiece material, but in a non-SP domain either due to:

1. The strain rate being above the traditional SP window

2. A non-ideal initial microstructure

3. The forming temperature being below that which is ideal for SP

However, seen from a mechanics point of view there is no clear mathematical criteria or experimental methodology that enables the construction of the boundaries of the SP domain (such as a yield surface) in a definitive way. It might be argued that the strain rate sensitivity might be used to define such a boundary, however, whether this is sufficient in itself is doubtful. The fact is that, as Fig. 1, demonstrates, $m$ will often vary as a function of strain rate and temperature.

From a manufacturing simulation point of view what do we need from a good process model? We need an optimised pressure versus time relationship for the forming cycle that is designed to minimise the forming time while maintaining an optimum strain distribution within the part. So, there is a need to be able to model a hot forming process and be able to map across all regions of the Stress / Strain rate space. To do this requires a model that can shift from a creep dominated regime, through SP proper, into a slip dominated regime. The model needs to be constructed in such a way that it can continuously re-balance the relevant form of the controlling algorithm to adjust appropriately as the localised deformation rate (and potentially temperature) vary.

\section{Analysis of SP domain}

As has been noted, the majority of investigators focus on the modification of the constitutive models of ideal SP, proving them with some simple sheet metal forming examples and generally do not explore the question of the range of validity of these models. However, in some recently published papers $[6,7]$ there has been a move to recognise that even using carefully calibrated models the results obtained from real forming operations can differ from predictions. From the viewpoint of FEM simulation and the correct mechanical description of material rheology, finding the range of applicability of SP constitutive models may be more important than finding new mathematical approximations. Mathematically, constitutive models of pure SP represent a situation 
where the flow stress is often almost constant and the material behaves similarly to a viscous liquid i.e. it does not depend on loading history. However, in the superplastic-like domain the behaviour of the material becomes more and more rheologically complex; the constitutive equations cease to be history independent, and the constitutive model takes a functional form.

Unfortunately, the establishment of the exact mathematical criteria for detecting the boundaries of the SP domain is not a trivial task. The difficulties are related in some degree with the nature of $\mathrm{SP}$ - it is not a threshold type phenomenon, which takes place when some particular microstructural mechanism suddenly gets switched on (as in the transition between elasticity and plasticity) and ends by it switching off. Another difficulty is related with the absence of a single strict definition of SP accepted by both the mechanics and material disciplines. Some analysis of the different types of SP definition is given in [4]. Summarizing that, we can say that the majority of these definitions mix up three elements: the phenomenon, its background and its manifestation plus, additionally, the conditions providing it. We can attempt to separate them as follows. With regard to the phenomenon the material begins to behave similarly to a viscous liquid (with little hardening or softening) accompanied with the high stain rate sensitivity $(>0.3)$. The background to this would be the set of the microstructural mechanisms providing the above mentioned property. The manifestation is set of loading processes in which the phenomenon can be observed and studied experimentally in an unambiguous way. The conditions are that set of macroscopic conditions which enable the SP deformation mechanisms to be active and dominating. These would include as a minimum temperature, strain rate and microstructure, but could be extended to include other factors e.g. the stress state.

However, even this separation does not bring us closer to the purpose of stating the SP criteria, in a mathematical form. The strain rate sensitivity factor seems to be the main quantitative characteristic which can be used for this purpose, though its experimental determination is related to the differentiation of experimental data, and the methodology may not be robust. However, using a value of $m=0.3$ is a somewhat arbitrary measure; higher $m$ values do not necessarily guarantee a SP response.

Given the foregoing, an alternative approach can be pursued. The problem can be inverted. This approach would use a general visco-plastic model that captures the mechanical behaviour across all domains of deformation including the SP and SP-like. Then, the mathematical conditions at which the functional relation transfers into a function will provide a boundary of the SP domain, and maybe additionally some metrics can be obtained, to gauge "how far or close" we are located about ideal SP. Lastly it may be required to classify the states - ideal SP, SP-like and non-SP.

\section{Constitutive modelling}

a)

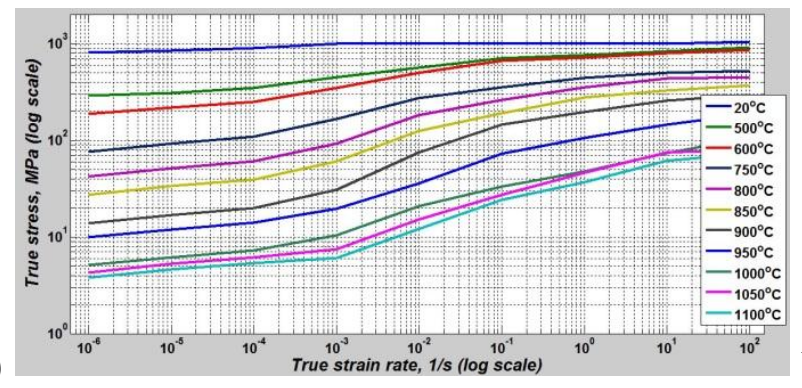

b)

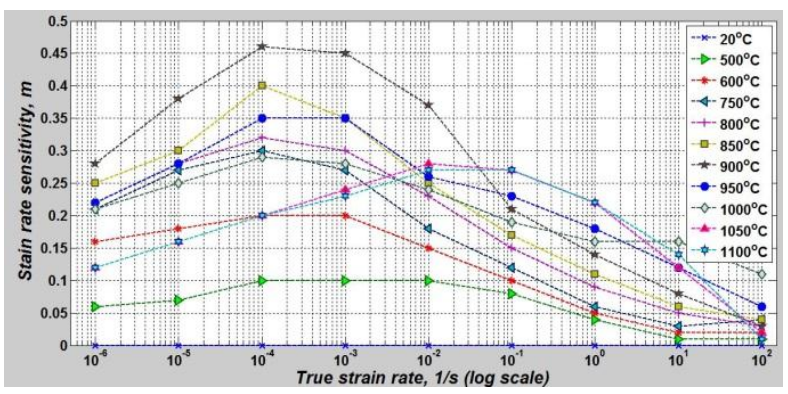

Figure 1. Typical mechanical data for Ti6Al4V with equiaxed fine-grained alpha of about 8 micron for the "initial part" of the stress-strain diagram $(\varepsilon \sim 0.1)$ for a wide range of temperatures covering $\alpha+\beta$ and $\beta$-zone: a) $\log \dot{\bar{\varepsilon}} \sim \log \sigma$ and b) strain rate sensitivity, $m$ (processed from data collected from [8-10]).

In Fig. 1 typical mechanical data is given for the behaviour of Ti-6Al-4V in a very wide range of static and quasi-static deformation as well as for a wide range of temperatures. As this covers a number of deformation processes (creep, SP, visco-plasticity, warm and hot working), based on 
different microstructural micromechanics, we do not strive to find any unified form of analytical constitutive model which will represent all the states. Instead of this we can try to cover the total parameter space of the process with a series of interlinked constitutive models that are locally accurate [11]. For example, the entire dataset given in Fig. 1(a) can't be described with either a power law or a hyperbolic sine function with fixed coefficients. However, in the small locality of any point the approximation, for example in the form in Eq. 1 will be acceptable:

$$
\sigma_{0}=A \dot{\varepsilon}^{m} \cdot \exp \left(\frac{Q}{R T}\right)
$$

Clearly, the values of $A, m$ and $Q$ will be different for each point. In Fig. 1(b), it can be seen how the rate sensitivity varies from point to point; more data on the variation of $m$ and $Q$ can be found in [12]. If a more accurate approximation is required, the discretization can be run to a finer scale, and intermediate data can be interpolated with different splines; this is easy to do directly in a FE simulation.

Another difficulty with the 'universal' constitutive model is related to the fact, that it is not sufficient just to find an approximation of the 'stress-surface'. This surface will itself change as a function of strain / microstructural development.

To try to resolve the problem with the transformation of the stress surface in the process of deformation, we can assume (similarly to the theories of plasticity), that the surface described above may be treated as a starting point. In terms of constructing this, a material can be characterised on the basis of an initial microstructure that will provide a SP response. Then a basic strain has to be chosen - it can be the smallest plastic strain that can be robustly measured in a tensile or compressive test. Here we indirectly assume that as this basic strain is small, the accumulated plastic energy is insufficient for any significant microstructure transformations to occur. By running a series of tests at this strain it is possible to develop a series of stress surfaces that effectively provide a series of starting points mapped out across temperature and strain rate space. The surface can be discretized and for each node in the parametric space of temperature and strain rate the values for the constants can be found. Alternatively, the stress values can be used directly and interpolated with a fitting function to any point of the space of the parameters.

It is then assumed that during deformation the microstructure of the material undergoes transformations which result in a modification of the stress surface. Mathematically it can be expressed as the product of the basic function to a modification factor, depending on the local microstructure transformation.

$$
\sigma=\sigma_{0}(T, \dot{\varepsilon}) \cdot \Lambda(\text { microstructure })
$$

The fact that the factor $\Lambda$ is written dependant only on microstructure doesn't mean that it is independent of the temperature and strain rate. The linkage is indirect via kinetic equations for the microstructural parameters.

It is evident, that the number of important microstructural parameters is large and normally different for different families of materials, so any attempt at their detailed description is no more promising than an attempt to develop a universal constitutive model. Theoretically, the number of internal variables can be unlimited provided that the kinetics can be described by known equations. But, as for the situation with crystal plasticity, this approach involves many non-evident assumptions which would make any derivative model difficult to calibrate and use. To bypass this, the idea proposed by Rabotnov [13] can be utilized. He has suggested the use of a limited set of internal variables, which will reflect the state of the structure, but which do not necessarily have a direct physical sense.

To illustrate this approach, we can examine a simple situation, when the word "microstructure" in Eq. 2 relates only to the some effective (micro-) structural unit (ESU), which may be the effective grain size, though in morphologically more complex alloys e.g. the two phase titanium alloys, might relate to the morphologic class of the microstructure. Then Eq. 2 may have for example the following shape: 


$$
\sigma=A \dot{\varepsilon}^{m} \cdot \exp \left(\frac{Q}{R T}\right) \cdot\left(\frac{D}{D_{0}}\right)^{k}
$$

Here, the value $D_{0}$ refers to that structural state for which the 'basic' stress surface was constructed. It should be mentioned, that obviously the power law form chosen for the modification factor is rather primitive and would not form the basis of a universal relationship on a wide range of loading conditions. This problem is resolved in the similar way as already described earlier for Eq. 1. This expression is nothing more than a local approximation of some complicated function. So, Eq. 3 has to be accompanied with the experimentally determined set of $k$ values for all the discretization nodes of the stress surface (similar to $A, m$ and $Q$ ).

Being supplemented with the kinetic equations for the ESU the mathematical constitutive model becomes closed and can be used for FEM simulations. A few brief comparisons of the results obtained with this approach for some SP and SP-like processes will be presented in the next section.

\section{Numerical simulations}

To illustrate some of the foregoing, an FEM simulation of a SP-like sheet forming process was conducted using different constitutive models for the same material and same loading conditions. The material and process was chosen similar to [1,2], however, to make the study clearer, it was limited with deviation from the SP range only in terms of strain rate; temperature and microstructure were set to be optimal $\left(900^{\circ} \mathrm{C}\right.$ - Fig. $2 \mathrm{~b}$, and $8 \mu \mathrm{m}$ fine grained microstructure) . To extend the range of the validity of the analysis, the data set was extended to include that cited by Seshacharyulu et al $[8,9,10]$; Fig. 1. The SP-like forming process consisted of two sequential operations: pre-shaping by deep drawing (Fig. 2a) followed by final gas forming (Fig. 2b). The second operation was assumed to be SP forming with a temperature of $900^{\circ} \mathrm{C}$ and pressure profile providing an average strain rate of $10^{-4}$ to $10^{-3} \mathrm{~s}^{-1}$. The pre-forming operation uses hot drawing of the sheet with a tool speed of about $5 \mathrm{~mm} / \mathrm{s}$. This generates strain rates between $10^{-2} \mathrm{~s}^{-1}$ to $5 \mathrm{~s}^{-1}$ i.e. falls into the regime of $S P$-like. To simplify the comparison of the models it was assumed that the total process is isothermal $\left(900^{\circ} \mathrm{C}\right)$ and microstructural transformations can be neglected.

The constitutive models, chosen for the comparison, are listed in the Table 1. Of course, this list is very limited and there exist more complex models, e.g. visco-plasticity models [14]. However, the main aim of the selection was to find the simplest possible approach for adequate simulation of $\mathrm{SP}+\mathrm{SP}-\mathrm{like}$ mixed processes, that was easy to apply for a wide range of engineering process design. It has to be mentioned here, that for the convenience and preciseness of using any constitutive model three things are of chief significance:

1. The known range of validity.

2. The known methodology for obtaining the parameters involved in the model.

3 . The robustness of the calibration process with regard to experimental uncertainties.

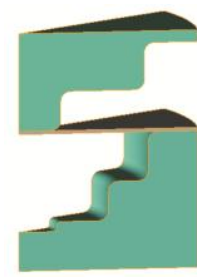

(a)

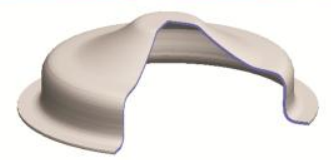

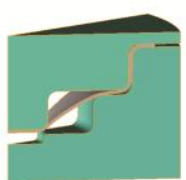

(b)
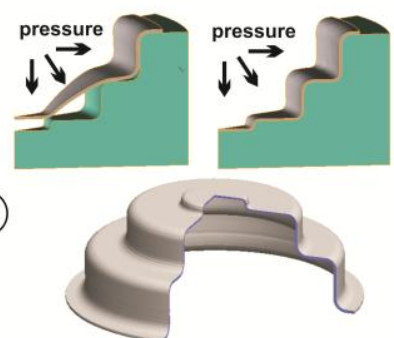

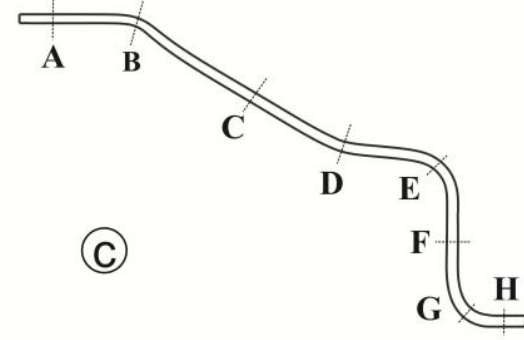

Fig. 2. The scheme of the simulated process with the shape of the part at the end of each stage: a) deep drawing at the pre-forming stage, b) pressure forming, c) scheme of thickness measurement. 
Table.1 Constitutive models of SP chosen for comparison. (All entities assumed to be dimensionless).

\begin{tabular}{|c|c|c|c|c|c|}
\hline & Authors & Equation(s) & $\begin{array}{l}\text { Variables \& } \\
\text { Parameters }\end{array}$ & $\begin{array}{l}\text { Calibration } \\
\left(\mathrm{T}=900^{\circ} \mathrm{C}\right)\end{array}$ & $\begin{array}{l}\text { Range of } \\
\text { validity }\end{array}$ \\
\hline 1 & $\begin{array}{l}\text { Backofen } \\
\text { (B) [4] }\end{array}$ & $\begin{array}{l}\dot{\varepsilon}=A \frac{\left(\sigma-\sigma_{0}\right)^{1 / m}}{d^{q}} \exp \left(-\frac{Q}{R T}\right) \\
\left.\sigma\right|_{\substack{d=\text { const } \\
T=\text { const }}}=A_{0} \dot{\varepsilon}^{m}\end{array}$ & $\begin{array}{l}A, m, q, Q, R \\
\sigma_{0}-\mathrm{const}\end{array}$ & $\begin{array}{l}\mathrm{A}_{0}=500 \\
\mathrm{~m}=0.45\end{array}$ & $\begin{array}{c}\frac{\text { Strain rate }}{\mathrm{R} 2} \\
\frac{\text { Temperature }}{?} \\
\frac{\text { Microstructure }}{?}\end{array}$ \\
\hline 2 & $\begin{array}{l}\text { Backofen- } \\
\text { Avery } \\
\text { (BA) [4] }\end{array}$ & $\begin{array}{l}\dot{\varepsilon}=A \frac{\sigma}{d^{q}}+B \sinh (\beta \sigma) \\
\left.\sigma\right|_{\substack{d=\text { const } \\
T=\text { const }}} \approx \frac{1}{\beta} \sinh ^{-1}\left(A_{0}+C \dot{\varepsilon}\right)\end{array}$ & $\begin{array}{l}A, q, B, \beta- \\
\text { const }\end{array}$ & $\begin{array}{l}\beta=0.08 \\
A_{0}=1 \\
C=10000\end{array}$ & $\begin{array}{c}\frac{\text { Strain rate }}{\mathrm{R} 1, \mathrm{R} 2, \mathrm{R} 3} \\
\frac{\text { Temperature }}{?} \\
\frac{\text { Microstructure }}{?}\end{array}$ \\
\hline 3 & $\begin{array}{l}\text { Smirnov } \\
\text { (S) [15] }\end{array}$ & $\sigma=\sigma_{s} \frac{\sigma_{0}+k \dot{\varepsilon}^{m}}{\sigma_{s}+k \dot{\varepsilon}^{m}}$ & $\begin{array}{l}\sigma_{0}, \sigma_{s}, k, m- \\
\text { const }\end{array}$ & $\begin{array}{l}\sigma_{0}=14 \\
\sigma_{\mathrm{s}}=258 \\
\mathrm{k}=500 \\
\mathrm{~m}=0.45\end{array}$ & $\begin{array}{c}\frac{\text { Strain rate }}{\mathrm{R} 1, \mathrm{R} 2, \mathrm{R} 3} \\
\frac{\text { Temperature }}{?} \\
\frac{\text { Microstructure }}{?}\end{array}$ \\
\hline 4 & $\begin{array}{l}\text { Bylya- } \\
\text { Blackwell } \\
- \text { Vasin } \\
(\mathrm{BBV}) \\
{[11]}\end{array}$ & $\begin{array}{l}\sigma=A \dot{\varepsilon}^{m} \cdot \exp \left(\frac{Q}{R T}\right) \cdot\left(\frac{D}{D_{0}}\right)^{k} \\
\dot{D}=\left\{\begin{array}{cc}t_{g} \exp \left(-\frac{Q}{R T}\right) & \text { if } \mathrm{D} \leq \mathrm{D}_{\mathrm{m}} \\
\left(\mathrm{c} \frac{\sigma}{D^{2}}+\mathrm{t}_{\mathrm{d}}\right) \cdot \exp \left(-\frac{Q}{R T}\right) & \text { if } \mathrm{D}_{\mathrm{m}} \leq D \leq D_{c r} \\
0, \mathrm{D}=0.7 \cdot D_{c r}, & \text { if } \mathrm{D} \geq \mathrm{D}_{c r}\end{array}\right. \\
D_{c r}=D_{1}+D_{c r 0} \exp \left(-B_{1} \int \sigma \cdot \dot{\varepsilon} \cdot d t\right)\end{array}$ & $\begin{array}{l}A=A(\dot{\varepsilon}, T) \\
m=m(\dot{\varepsilon}, T) \\
Q=Q(\dot{\varepsilon}, T) \\
k=k(\dot{\varepsilon}, T) \\
\text { Numerical } \\
\text { interpolation }\end{array}$ & $\begin{array}{l}\text { m - Fig. } 2 b \\
\text { A, Q [11] } \\
\text { k not used here }\end{array}$ & $\begin{array}{c}\frac{\text { Strain rate }}{\mathrm{R} 1, \mathrm{R} 2, \mathrm{R} 3} \\
\frac{\text { Temperature }}{20-1100^{\circ} \mathrm{C}} \\
\frac{\begin{array}{c}\text { Microstructure } \\
1-1000 \mu \mathrm{m}\end{array}}{}\end{array}$ \\
\hline
\end{tabular}

All the models listed in the table were calibrated using the experimental data for fine-grained Ti6Al-4V, shown in Fig.1. Unfortunately, for models 1-3 their calibration with the same set of constants for the range of temperatures is not trivial (if possible at all) even if temperature is directly involved, as per model 1. For this reason, we have limited our investigation with the SPlike processes that deviate from pure SP only in terms of strain rate (temperature and microstructure - assumed to be SP grade - and their changes are neglected). Calibration for a temperature of $900^{\circ} \mathrm{C}$ is presented in Fig. 3. All the models were programmed as user-functions using the metal forming software; QForm8, and used for the simulation of the process described in Fig. 3. It has to be mentioned that the main attraction of model 1 is the ability to find its constants directly analytically from experiments. The methodology for this is well established. For the rest of the models the question of proper calibration is not trivial [16]. Many authors [6, 7] are using numerical minimisation methods to adjust (tune) the model parameters. The results of this process depend significantly on the algorithms used, and are very uncertain if a large number of parameters are used in the model. They are also very sensitive to the accuracy of input data.

The results of the pre-forming operation (Fig. 2), calculated for the calibrated models are shown in the Fig. 3b. Though, as can be seen from Fig.3a, the approximations of the last three models are very close, some difference in the resulting shapes is visible (the shape of corner G, parts BD and EF). However, as can be seen from the table of thicknesses (Fig.3b), the predictions remain reasonably close. If higher accuracy is not required, in principle all simulations may be accepted, though they will lead to different pressure profiles and result in higher thickness deviation (and 
error) in the second operation. This result may be interpreted as indicating that this process is not that far from SP forming and can be treated as SP-like.

a.
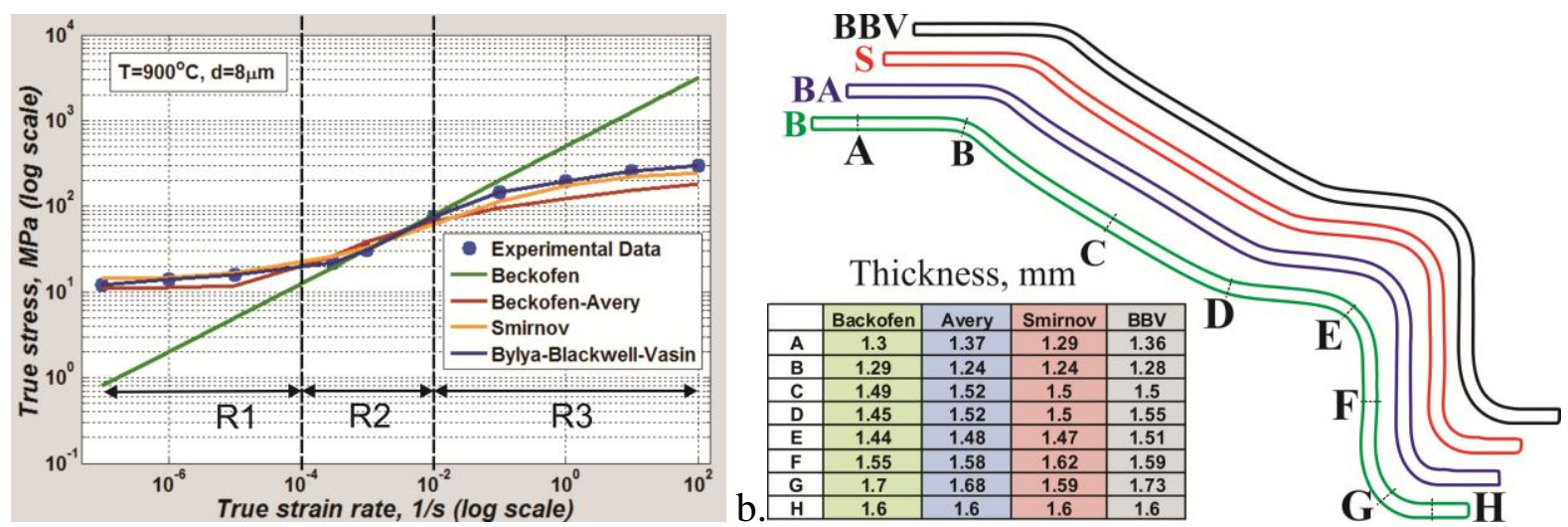

Fig. 3.a) Calibration chart for models listed in the Table 1. Material is Ti-6Al-4V, equiaxed $\sim 8 \mu \mathrm{m}$ grain size, temperature $900^{\circ} \mathrm{C}, \mathrm{b}$ ) the shape and thickness of the part after pre-forming operation, calculated using different models.

Another situation is observed while we move further away from the SP domain. The difference between models can be more clearly seen if in the first deep drawing operation the outer part of the formed blank is kept fixed; Fig.4. In reality, if the outer edge of the blank were fixed in this way the first operation likely could not be performed due to failure of the material. However, as can be seen from Fig. 4, some models (such as Backofen and Smirnov) don't predict any failure, another ones (Backofen-Avery and Bylya-Blackwell-Vasin) predict failure in different parts of the sheet. Such differences in the results of simulation are related to the fact that in contrast to the previous example, conditions of this process are further away from ideal SP, so the behaviour of the material is more complex and only fitting the sigmoidal curve (Fig.1, Fig.3a) is insufficient for obtaining adequate results.

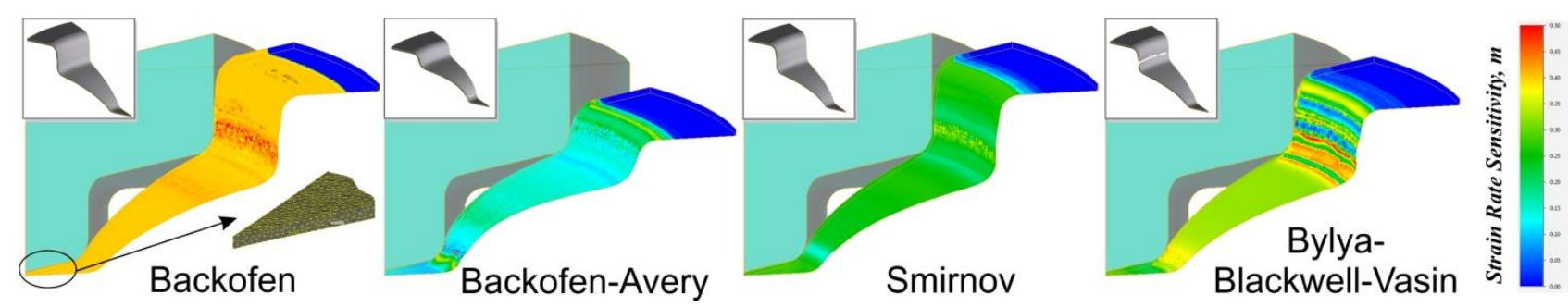

Fig. 4.The results of simulation of the deep-drawing operation with the perimeter of the sheet fixed. The simulation is made with the 3D triangle elements, with minimum 4 elements per thickness.

\section{Summary}

1. Technologies which are using SP have good prospects, but the choice of this technology is expensive and generally used only where other manufacturing techniques cannot be used. In the real SP process optimal conditions are generally not obtained across the workpiece throughout the process.

2. There are many models to represent SP, however they tend to be applicable in a fairly narrow range of operating conditions and if the local deformation parameters (temperature, strain rate) vary outside of the window of their validity the models may lead to erroneous predictions.

3. To describe near-SP along with SP conditions it is quite effective to use constitutive equations with internal variables which reflect the state of the microstructure though these variables may not be tracked against specific microstructural factors e.g. grain size. 
4. Outside of the optimal SP forming window the significance of the loading history becomes more important since more mechanisms of deformation are involved. It is not necessary to represent this with only one type of constitutive equation. For different areas of the deformation space different types of constitutive equation can be used as is sometimes done in the theory of plasticity.

\section{References}

[1] M. Guo, J. Liu, M.J Tan, B. Chua, Microstructure evolution in Ti-6Al-4V during superplasticlike forming, Procedia Engineering, Vol.81 (2014) pp1000-1005.

[2] J. Liu, J. Edberg, M.J. Tan, L.E. Lindgren, S. Castagne, A.E.W. Jarfours, Finite element modelling of superplastic-like forming using a dislocation density base model for AA5083, Modelling Simul. Mater. Sci. Eng. Vol.21 (2013) 025006, pp1-24

[3] O.V. Sosnin, B.V. Gorev, A.A. Ratnichkin, Mechanics of Superplasticity and its Relationship with High Temperature Creep. Siberian Magazine of Physics and Technology, Vol.4, (1993) pp.15-23. (in Russian).

[4] K.A. Padmanabhan, R.A. Vasin, F.U. Enikeev, Superplastic flow: phenomenology and mechanics, Pub. Springer Verlag, (2001).

[5] J. Lin, F.P.E. Dunne, Modelling grain growth evolution and necking in superplastic blow forming, Intl. Journal Mech. Sci. 43 (2001) pp.595-600.

[6] E. M. Taleff, L.G. Hector Jr., J. R. Bradley, R. Verma, P. E. Krajewski: Acta Mater. Vol.57 (2009) pp. 2812-2822.

[7] M. Albakri, F. Abu-Farha, M. Khraisheh: Int. J. Mech. Sci. Vol. 66 (2013) pp. 55-66

[8] T. Seshacharyulu, S.C. Medeiros, W.G. Frazier, Y.V.R.K. Prasad, Hot working of commercial Ti-6Al $-4 \mathrm{~V}$ with an equiaxed $\alpha+\beta$ microstructure: materials modeling considerations, Materials Science and Engineering A248 (2000) pp.184-194.

[9] A.K. Ghosh, C.H. Hamilton, Mechanical behavior and hardening characteristics of a superplastic Ti-6Al-4V alloy, Metall. Trans. A. Vol.10A (1979) pp.699-706.

[10] I.F. Anoshkin, G.A. Bochvar, I.S. Livanov, V.A. Pol'kin, V.I. Moiseev, Metallography of Titanium Alloys (1980), Moscow, Metallurgy. 464p.

[11] O.I.Bylya, M.K Sarangi, N.V.Ovchinnikova, R.A.Vasin, E.B.Yakushina, P.L.Blackwell, IOP Conf. Series: Materials Science and Engineering, 63012033 (2014)

[12] O.I.Bylya, B.K.Pradhan, E.B.Yakushina, P.L.Blackwell, Modelling of active transformation of microstructure of two-phase Ti alloys during hot working // Letters on Materials 4 (2), (2014) pp.124-129.

[13] Y.U. Rabotnov, Creep Problems in Structural Members, (North-Holland Series in Applied Mathematics and Mechanics.). Pub. Amsterdam/London.North-Holland,1969.

[14] Y.Q Wu, K.S. Zhang, Numerical and experimental analysis of superplastic-like uniaxial tensile necking of coarse-grained LY-12, Mechanics of Materials 35 (2003) pp.1127-1138

[15] Smirnov, O.M, Metal Working in a Superplastic State. Mashinostroyenie, Moscow (1979) (in Russian).

[16] A. Aksenova, E.N. Chumachenko, A.V. Kolesnikov, S.A. Osipov Determination of optimal gas forming conditions from free bulging tests at constant pressure, Journal of Materials Processing Technology 217 (2015) pp.158-164.

Acknowledgement: RAV would like to acknowledge funding from Grant RFBR N 15-08-04281 